\title{
El papel del maestro en la transformación educativa ante un escenario de incertidumbre: Reflexiones desde y para México
}

\author{
Cruz Eréndida Vidaña Dávila ${ }^{1}$, Carlos Rodríguez Ramírez ${ }^{2}$, José de Jesús Paredes ${ }^{3}$
}

\section{Resumen}

El mundo actual tiene un carácter marcadamente dinámico, por lo que el Sistema Educativo Mexicano (SEM) en su conjunto, pero particularmente las escuelas formadoras de docentes y las prácticas de enseñanza en el aula, deben transformarse para hacer frente a esa realidad. Se reconoce que el unico agente indefectible que puede generar ese cambio es el docente que abrace una pedagogía crítica y que sea conocedor de la ciencia, la pedagogía y la filosofía para que ejerza una práctica reflexiva. El presente trabajo presenta una revisión teórico conceptual de la necesidad de docentes críticos transformadores en la realidad mexicana, empleando referentes como Freire y analizando también algunos elementos de la Nueva Escuela Mexicana (NEM). Un docente crítico y transformador se forja en las escuelas normales, desarrollando y potenciando lo que un profesor que asuma ese rol debe tener. Esas cualidades no se logran desde la simple experiencia, es necesario que se allegue de referentes de orden filosófico que le permitan, primero, reflexionar sobre el impacto de buenas prácticas de enseñanza, luego, tomar acción como agente de cambio. Además, debe abonar a la construcción de una filosofía emergente que le ayude a resolver problemas reales, como el generado por la COVID-19, donde se observó que la autoridad educativa dejó de lado esta reflexión filosófica y partió de concepciones rígidas, cerradas y tradicionalistas de currículo.

Palabras clave: Transformar; pedagogía dominante; pedagogía crítica; violencia simbólica; reflexión de la práctica

\section{The role of the teacher in educational transformation in a scenario of uncertainty: Reflections from and for Mexico}

\begin{abstract}
Today's world has a markedly Dynamic character which demands the Mexican educational system as a whole, but specifically the schools where the new teachers are formed, as well as the classroom teaching practices, to be transformed to face that reality. We recognize the only unfailing agent that is able to generate that change is the teacher who embraces a critical pedagogy and who also knows the science, pedagogy and philosophy to exert a reflective practice. A critical and transforming teacher is formed in teacher education schools, developing and improving what a teacher who takes that role must have. These qualities are not attained by means of simple experience, what is truly necessary is that he or she obtains philosophic referents that allows him or her, first, to reflect on the impact of good teaching practices, then, become an agent of change. Additionally, he or she must contribute to the construction of an emerging philosophy that would help bim or her solve real problems, such as the one that COVID-19 generated, where we witnessed that the educational authorities left aside this philosophic reflection by sustaining rigid, unwavering, and traditional conceptions of the curriculum.
\end{abstract}

\footnotetext{
${ }^{1}$ Cruz Eréndida Vidaña Dávila, Docente del Centro de Actualización del Magisterio, Zacatecas e integrante del Grupo de interés "Práctica Docente en la Formación de profesores, Mexico. Correo electrónico: erendida@camzac.edu.mx.

${ }^{2}$ Carlos Rodríguez Ramírez, Docente del Centro de Actualización del Magisterio, Zacatecas e integrante del Grupo de interés "Práctica Docente en la Formación de profesores, Mexico. Correo electrónico: admin@tplondon.com.

${ }^{3}$ José de Jesús Paredes, Docente del Centro de Actualización del Magisterio, Zacatecas e integrante del Grupo de interés "Práctica Docente en la Formación de profesores, Mexico. Correo electrónico: admin@tplondon.com.
} 
70 El papel del maestro en la transformación educativa ante un escenario de incertidumbre. Reflexiones desde y para México

Keywords: Transform; dominant pedagogy; critical pedagpgy; symbolic violence; reflection on practice teaching

\section{Introducción}

La tesis central que sustenta este trabajo sostiene que hay un imperativo insoslayable: la educación debe transformarse sin demora para responder a las demandas de una sociedad que hoy exige el reconocimiento de las minorías y, sobre todo, que coadyuve a la formación de ciudadanos libres, pensantes, reflexivos, críticos, analíticos, propositivos, empáticos y capaces de incidir favorablemente en la resolución de los problemas actuales de los ámbitos local y global, para que puedan incidir en la mejora dela realidad, pero siempre regidos por principios éticos sólidos. El único agente indefectible que creemos que puede generar ese cambio es el docente, pero no cualquier docente, sino aquél que abrace una pedagogía crítica, que le distingan conocimientos pedagógicos y disciplinares profundos, que cuente con las habilidades intelectuales de orden superior y con los mismos valores éticos que está llamado a favorecer en sus educandos. Con base en lo expuesto es que este texto tiene el propósito de propiciar la reflexión en el lector sobre la necesidad de transformar la educación para responder a una realidad que, por su carácter dinámico, desde hace décadas exige un cambio de rumbo en las formas tradicionales de educar.

Es impostergable superar la visión centrada en la mera transmisión de contenidos, a veces irrelevantes, y orientada hacia la aculturación de la población a fin de ceñirla a una forma de pensar y actuar hegemónica (Tünnerman, 2008). El escenario actual se caracteriza por la incertidumbre (Barrón, 2020) y porque como lo mencionaba Zygmunt Bauman (2008), los valores y las certezas que la sociedad había instituido como dogmas, hoy han desaparecido; las verdades se vuelven líquidas y abandonan su carácter estático que la tradición les había otorgado. ¿Acaso habrá transformación más necesaria que la que debe hacerse en el ámbito educativo?

Este escrito se ha organizado en tres apartados, en donde se ponen de manifiesto puntos críticos que aquejan la enseñanza y el aprendizaje: el primero de ellos, titulado "La pandemia del COVID-19, escenario de incertidumbre para repensar la educación”, reflexiona sobre los retos que impuso al Sistema Educativo Mexicano (SEM) la situación de aislamiento social y la forma tan rígida y tradicionalista en la que la autoridad y actores educativos reaccionaron ante la emergencia sanitaria mundial. El segundo: "La formación filosófica del profesor como agente de transformación y cambio", habla de la trascendental tarea de formar al nuevo docente que asuma el rol activo que la transformación educativa requiere, y el tercero, "Los profesores que demanda la sociedad del siglo XXI", analiza las características que el docente de nuestros tiempos debe poseer para responder a las necesidades de la sociedad.

\section{La pandemia del COVID-19, escenario de incertidumbre para repensar la educación}

En el mes de marzo del año 2020, la vida de los mexicanos dio un giro súbito e inesperado: se impuso el aislamiento social como primera medida de contención dela pandemia causada por la COVID-19. Este aislamiento provocó que todos los planteles educativos a lo largo y ancho del país cerraran sus puertas y que el formato de clases presenciales diera paso a una nueva modalidad: la enseñanza a distancia. 
Para afrontar la nueva realidad, toda vez que las pandemias son de larga duración y modifican sustancialmente las formas en que los seres humanos nos relacionamos no sólo con nuestros semejantes, sino con cada aspecto de nuestro entorno, una de las primeras acciones que emprendió el Gobierno Federal fue abrir las puertas a empresas como 'Google for education', mismas que capacitaron a los docentes en el uso de sus herramientas tecnológicas, sobre todo las que facilitan la enseñanza virtual. Posteriormente, la Secretaría de Educación Pública (SEP), diseñó un programa de apoyo a la educación obligatoria llamado "Aprende en casa", que consistió en emisiones televisivas en las que un profesor exponía el contenido de la asignatura en cuestión de manera concisa, mientras que los alumnos, tras ver y escuchar el programa, debían responder a una serie de preguntas (Díaz-Barriga, 2020). Este proceder también corresponde al paradigma tradicionalista que urge erradicar.

Las apariciones de la autoridad educativa en distintas redes y medios de comunicación fueron constantes durante el período de aislamiento. En sus discursos resaltaba la importancia de "Salvar el ciclo escolar" y que, para lograrlo, los alumnos deberían revisar los contenidos propuestos en el currículo. Estas declaraciones permiten apreciar nítidamente la visión rígida y cerrada que la SEP tenía de currículo, toda vez que esta dependencia del gobierno federal mantuvo una mirada centralizada y vertical que orientó el trabajo de manera unidireccional (Chehaibar, 2020). El énfasis se puso en la revisión de contenidos disciplinarios alejados de la realidad (Barrón, 2020) que buscaban a toda costa lograr los aprendizajes esperados (DíazBarriga, 2020)

Las acciones emprendidas por la SEP, si bien se hicieron bienintencionadamente, aumentaron las desigualdades sociales debido a que, entre otros desaciertos, no se consideraron las condiciones dispares de la población escolar de acceso a las plataformas digitales, ni tampoco se valoró la disponibilidad delos recursos, sobre todo tecnológicos, con los que contaban maestros y alumnos ante el reto de la educación a distancia (Díaz-Barriga, 2020).No basar las acciones en estos aspectos de la realidad generó que las estrategias para habilitar a los profesores en el manejo de herramientas de Google, resultara poco fructífero.

Por otro lado, ante ese nuevo escenario de incertidumbre, la escuela respondió con viejas concepciones y desaprovechó la oportunidad de repensarse y preguntarse qué escuela se necesita hoy y para qué sociedad (Plá, 2020). Esta situación inédita dejó al descubierto el pensamiento tradicionalista, pasivo y fuertemente tecnocrático con el que se enseña, pues en lugar de flexibilizar el currículo a partir de proyectos integradores que promovieran la reflexión y el aprendizaje de la realidad (Díaz-Barriga, 2020), los esfuerzos educativos se enfocaron en la observancia del currículo desde una perspectiva rígida que llevó a padres de familia, alumnos y docentes a convertirse en trabajadores de tiempo completo y a invadir los espacios personales con actividades académicas (Barrón, 2020). Dichas actividades bajo el modelo de cumplir, hacer y enviar tareas aisladas y dirigidas cuyos aprendizajes generaron principalmente conocimientos frágiles en los alumnos.

La pandemia invitaba a repensar el sentido de la institución escolar y la naturaleza y pertinencia del currículo formal. Era el momento propicio para desescolarizar la educación y de alguna forma, invertir la manera de visualizar y aplicar el currículo, es decir, dejar de pensar en organizar el contenido desde las disciplinas y ponerlo al servicio de las necesidades reales de los alumnos, como atinadamente lo señala Ángel Díaz-Barriga (2020).Se trata de desaprender para aprender, lo que indudablemente debería llevarnos a replantear las finalidades del SEM 
72 El papel del maestro en la transformación educativa ante un escenario de incertidumbre. Reflexiones desde y para México

porque el diagnóstico estaría centrado en un contexto dinámico que demanda nuevos paradigmas educativos (Plá, 2020).

La oportunidad invaluable que generó la pandemia para repensar la educación ha sido desaprovechada por todo el sector educativo debido a los paradigmas filosóficos que sostiene la autoridad educativa, y sobre cuya base ellos toman decisiones. Al respecto, Picardo (2002) afirma que cualquier política y/o acto educativo engloba una perspectiva filosófica. En este caso, los posicionamientos mostraron que la realidad percibida por el sistema educativo es concebida como estática e inamovible, sustentada en visiones positivistas o empiristas, en las que el sujeto solo cumple un papel pasivo en la construcción del conocimiento, por lo que transformar y repensar la educación desde esta nueva realidad resulta prácticamente imposible.

Al comprender la realidad desde un posicionamiento filosófico rígido aparece una gran dificultad para transformar y repensar la educación, tal como lo establece Zweig citado por Delgado (2020):

"La tradición es una muralla de piedra hecha de pasados que rodea el presente: quien quiere ir hacia el futuro tiene que saltarla, pues la naturaleza no tolera altos en el camino del conocimiento [...] parece defender el orden, sin embargo, sólo ama a quien la destruye para crear un orden nuevo" (p. 188)

La respuesta del sistema educativo mexicano ante la pandemia fue desatinada, incapaz de saltar los muros de la tradición que se sostenían en los rituales presenciales de la escuela. La humanidad y en particular el sistema educativo redujo la educación al cumplimiento de un horario definido dentro de una institución dedicada a revisar una lista de contenidos inamovibles (Díaz-Barriga, 2020), dejando de lado la esencia y el significado de educar y formar a los hombres que conformarán la sociedad del futuro. Los efectos de esta visión son y serán desalentadores.

\section{La formación filosófica del profesor como agente de transformación y cambio}

El escenario de incertidumbre generado por la pandemia revela la imperiosa necesidad de un nuevo profesor que repiense su papel como un agente de transformación, capaz de modificar la función reproductora de violencia simbólica y contribuya a disminuir la desigualdad social a la que ha contribuido inconscientemente (Lucio et al, 2018).El docente que se necesita para el contexto de la modernidad líquida debe reflexionar en torno a varios planteamientos básicos que Freire reconoce como aspectos centrales para el desarrollo del pensamiento crítico (Rondón y Páez, 2018), además de ser capaz de asumir actitudes de humildad ante lo que no sabe, valentía para llevar en alto la profesión docente, aunque la sociedad la desestime; amor por el acto de educar, tolerancia y paciencia para convivir, entender y aprender de las situaciones y de las personas diferentes, y alegría por llevar a cabo la práctica educativa. De igual forma, debe desarrollar habilidades como el estudio, la lectura, el enseñar a leer, dialogar y enseñar a escuchar y tomar decisiones (Rondón y Páez, 2018).

La formación de un docente crítico y transformador no se logra desde la experiencia o la cotidianeidad y mucho menos si ésta no se somete al análisis permanente, es necesario que el profesor se apropie de una serie de referentes filosóficos que lo lleven a reflexionar desde un ángulo inédito su función dentro de la educación. Para ello, es importante que entienda que la filosofía no es un campo del saber alejado de su tarea, sino que aquellas definiciones dadas 
por los pensadores clásicos se relacionan estrechamente con su quehacer cotidiano. Al profesor le sirven concepciones como la de Platón, quien sobre filosofía afirmaba que era "la ciencia de las ideas" (Tünnerman, 2008); como la de Descartes, quien la veía como la ciencia que investiga los principios de todas las ciencias; como la de Wolf, para quien era la ciencia de las cosas posibles y de los fundamentos de su posibilidad, o como la pensaba Husserl, quien la asoció con "la ciencia sin supuestos fundada en el método fenomenológico" (Tünnerman, 2008, pág. 29).

Ante una ciencia definida de tantas maneras, Picardo (2002) especifica que existen diversas formas de hacer filosofía, lo que significa que su objeto de estudio no está dado como en otras ciencias, más bien se hace o se busca, por lo tanto, el papel del filósofo es muy importante, pues establece su propio objeto, sistematización y metodología. Pero, ¿qué significa filosofar? consiste el develar, descubrir la realidad para aprehender lo esencial de ella, implica cuestionarse sobre lo que se considera establecido o como una realidad dada. En este sentido, el filósofo no es aquel que sabe o dice saber, sino el que reconoce que no lo sabe y busca encontrar la verdad, es decir, ama el saber y va tras él incesantemente.

Filosofar no es una tarea exclusiva de expertos, sino que debe ser un quehacer que el maestro desarrolle a diario en su tarea de educar a fin de comprender el significado de sus acciones, y cuestionarse sobre las realidades estáticas que nos hacen reaccionar con los mismos modelos curriculares rígidos ante un escenario de incertidumbre y cambios constantes.

En este contexto surge la necesidad de definir a la filosofía de la educación, entendida como la disciplina que estudia el comportamiento de la educación a la luz de las leyes que regulan el desarrollo de la sociedad humana, las diferentes concepciones del mundo y las formas como ellas conciben el hecho educativo (Dante, 2010). Además, se considera como el saber teleológico de la educación, es decir, se refiere a los fines: ¿Para qué se educa?, donde se define el tipo de hombre a formar. Por su parte, Ratio (2014) entiende a la filosofía de la educación como el deseo del saber para alimentar a las personas, es decir, muestra el derrotero de aquello que es necesario cumplir para formar al hombre y a la sociedad a la que se aspira.

El docente debe partir de un razonamiento filosófico para comprender la tarea que implica formar a los hombres del mañana, para lo cual resulta necesario que se pregunte: ¿qué es la educación? y comprenda que este concepto no tiene un significado único, ni total, pues depende de la corriente filosófica y el momento histórico desde el que se defina. Al respecto, Tünnerman (2008) distingue las siguientes concepciones: desde la corriente racionalista la educación se entiende como un medio para llegar a la verdad, el positivismo la concibe como un recurso para obtener conocimientos a partir del método científico, el pragmatismo la percibe como la actividad al servicio de la resolución de problemas inmediatos del "aquí y el ahora", subordina el pensamiento a la acción; y desde el marxismo o corriente dialéctica se reconoce como un medio de reflexión y transformación social.

El razonamiento filosófico que el docente desarrolle para cumplir con su papel de educar a las futuras generaciones corresponde a una postura filosófica desde la que comprende la educación, lo que hace necesario que tome conciencia hacia donde se orienta su pensamiento, a fin de identificar cuáles son los fines que persigue, esto le ayudará a transformar su realidad, pues como lo establece Freire (citado por Morales en Lucio et al, 2020), la reflexión de la práctica docente es importante para comprender desde qué referentes se actúa y cómo se puede generar un cambio en pro de los alumnos y de las minorías olvidadas. 
74 El papel del maestro en la transformación educativa ante un escenario de incertidumbre. Reflexiones desde y para México

A fin de lograr que la reflexión de la práctica realizada por el maestro tenga un verdadero impacto en la transformación de la realidad, es necesario que el docente sepa que existen distintas aportaciones filosóficas que le ayudan a comprender su labor educativa y que unas le proporcionarán mejores resultados educativos según el contexto y sociedad en que se encuentra, en este sentido, un profesor que desea mejorar su entorno debe conocer toda esa gama de posibilidades de acercarse al conocimiento de la realidad, por ejemplo, el método mayéutico de Sócrates y comprender que el papel del maestro consiste en problematizar al alumno para conducirlo a la verdad (Tünnermann, 2008, p. 38).De igual forma, debe revisar las aportaciones de Platón sobre la democracia, la organización de polis, el mito de la caverna y la formación del sujeto (Picardo y Escobar, 2002). La duda metódica de Descartes, los pensamientos de John Locke en torno a la educación, en la que explicaba cómo surgían las ideas; la postura empirista de David Hume (Picardo y Escobar, 2002), la visión educativa de Jean Jacques Rousseau, visto como el "liberador del niño" y como el "padre" de la educación progresista moderna, la fenomenología del espíritu de Hegel, los postulados de Juan Amos Comenio expuestos en su obra de "Didáctica Magna", la teoría Marxista de la educación que se presenta como forma y método de reintegración del hombre en el trabajo, la "teoría crítica de Frankfurt, las aportaciones de Piaget, Vygotsky, Ausubel y Chomsky, entre muchas otras ideas.

El razonamiento filosófico del profesor debe partir de la concepción de hombre que éste sustente, en la que se reconoce al sujeto como un ser pensante, capaz de transformar su propia realidad y generar conocimiento en un ir y venir entre los conceptos y las ideas, además de establecer una relación recíproca con el objeto de estudio en el que ambos se modifican mutuamente. También debe tener en cuenta los principios de la filosofía de la educación, como el de la educabilidad, la individualización, socialización, intuición el principio del juego, la creatividad, criticidad, cooperación, adecuación y de calidad total, (Dante, 2010), esto le ayudará a comprender que tiene una tarea trascendental en la formación de la sociedad, pues es el responsable de generar los espacios para que el niño se convierta en un ciudadano crítico, autónomo y capaz de transformar su realidad.

La visión filosófica que el docente construya a partir de distintos elementos como la comprensión del impacto de la filosofía en la educación, las aportaciones históricas de grandes filósofos, la reflexión sobre el hombre y los principios y los fines de la educación, le ayudarán a comprender lo que ocurre en su aula desde una visión holista e integral y, en consecuencia, permitirá entender el impacto de sus acciones y el tipo de ciudadano que está formando.

Es importante que esta visión filosófica no se reduzca a la contemplación del hecho y a una comprensión teórica: es necesario que se reflexione en torno a los problemas reales que se presentan en las escuelas. Al respecto, Arteaga (2014) plantea una propuesta muy interesante para resolver los problemas de los sistemas educativos de los países latinoamericanos, la cual consiste en desarrollar una filosofía emergente que ayude a los actores educativos y a la sociedad en general a reflexionar y repensar los problemas reales de su entorno.

Arteaga (2014) considera que ante una realidad cambiante es necesario que se construya una filosofía emergente que permita reflexionar desde diversas cosmovisiones culturales, formas de consciencia social y de las propias minorías "nuevos caminos metodológicos y visiones prospectivas más amplias, multidimensionales, de mayor comprensión y aproximación a fin de solucionar los problemas reales" (p. 183).Esto significa que esta filosofía se encuentra en 
construcción, pues debe partir desde y para la realidad; además, prioriza la acción sobre la contemplación, es decir, se trata de comprender para mejorar.

La filosofía emergente se convertirá así en un saber que el profesor posee, el cual no se limita a una disciplina o especialidad, sino que es multidimensional, capaz de comprender los fenómenos en contextos complejos y cambiantes (Arteaga, 2014). Este tipo de reflexiones ayudará a que el profesor se detenga a pensar el sentido de la educación y de su propia práctica a fin de valorar qué tan pertinentes son ante las necesidades de una realidad que se transforma de forma vertiginosa.

Este razonamiento que hará el profesor desde la filosofía emergente, lo ayudará a comprender el fenómeno educativo, dentro del fenómeno social y enfrentar con mejores herramientas conceptuales y sobre todo fundamentadas cualquier situación que pueda aparecer extraordinaria, como es el caso del fenómeno mundial de salud que actualmente afecta a la humanidad, lo que le permitirá ver que la educación a distancia generada por la pandemia no puede responderse con los mismos formatos de la educación presencial, ni puede mantener la esperanza de que las cosas volverán a la normalidad, pues sin duda, esta situación inédita demostró que la educación debe ser reformada desde sus cimientos a fin de que realmente responda a su función de educar y formar a los ciudadanos.

\section{Los profesores que demanda la sociedad del siglo XXI}

La modernidad líquida (Bauman, 2008) en la que vivimos se caracteriza por una sucesión de cambios constantes, hecho que demuestra quela formación de profesores no puede permanecer inalterada, en los mismos términos que lo ha hecho hasta ahora. Hoy, con mayor empeño, es necesario propiciar un cambio en las prácticas y el currículo de las instituciones de enseñanza, sobre todo el de las escuelas normales, con lo que se fomentará la formación de docentes críticos, capaces de incidir favorablemente en los contextos educativos y en la educación emancipadora de las minorías.

Hasta ahora, la educación se ha caracterizado por la instrumentación de una pedagogía dominante, misma que favorece los intereses de los más poderosos y acentúa las desigualdades sociales (Lucio et al, 2018), con lo que la escuela se convierte en reproductora de la violencia simbólica, pues transpone los mecanismos de segregación social en las aulas. Una de las instancias en las que este fenómeno se ve con toda nitidez es el de la evaluación, práctica que es utilizada como un medio de certificación, con lo que se acentúa la división de los más hábiles sobre los más débiles y de los que han tenido condiciones de vida a favor sobre los más vulnerables. La formación tradicional de profesores no los condiciona para ejercer dicha violencia, sino que los prepara "concienzudamente para hacer cumplir normas, métodos y disciplinas, con el propósito de reproducir los modelos sociales y económicos fielmente" (Lucio et al, 2018).

La pedagogía dominante genera una visión deshumanizadora de la profesión docente: los profesores dejan de lado la tarea de educar a personas pensantes que se integrarán a la sociedad y los comienzan a ver desde la perspectiva de la educación bancaria (Freire, 1985) basada en una enseñanza repetitiva de palabras inertes provenientes de la cultura dominante, en la cual se utilizan estrategias como la narración, se mitifica la realidad y se desconoce a la persona como ente histórico (Lucio et al, 2018). 
76 El papel del maestro en la transformación educativa ante un escenario de incertidumbre. Reflexiones desde y para México

La deshumanización de la profesión docente, la pedagogía dominante y la educación bancaria (Freire, 1985) son fenómenos desde los que se han diseñado e implementado los currículos de formación docente, generando que los profesores recién egresados se conviertan en reproductores de la violencia simbólica de la que a veces, ni ellos mismos son conscientes, tal como lo señala Bourdieu (citado en Lucioet al 2018), es así como se forma un sistema educativo poco crítico, que repite patrones año con año y reacciona con los mismos paradigmas y formatos ante cualquier situación, como ocurrió en la pandemia generada por la COVID-19.

Ante un escenario incierto, conviene preguntarse qué profesores se necesita formar. Sin duda, Freire (citado en Lucioet al, 2018)tiene una respuesta muy certera: él considera que es necesario que los docentes se conviertan en pensadores y problematizadores de su realidad, para ello, deben partir de un razonamiento filosófico que les permita preguntarse cuestiones básicas que tradicionalmente se han tomado como verdades acabadas sobre la vida, la organización social y la educación, por ejemplo: ¿qué es la realidad? En este sentido, el profesor debe cuestionar sus propias ideas a fin de llegar a la conclusión de que la realidad es una construcción dinámica en la que intervienen distintos factores y que caracterizarla dependerá del momento histórico desde el que se estudie.

Cuando el profesor comprende el carácter dinámico de la realidad, puede entender que él es un agente de cambio y contribuirá la transformación y mejoramiento de su entorno. En este sentido, la concepción de Freire sobre educación muestra la relación que se establece con la realidad, pues considera que ésta debe ocuparse de los temas que surgen de los problemas y necesidades que los oprimidos han identificado por sí mismos, de tal manera que la escuela debe ayudar a los hombres a percibir de una manera crítica su existencia en el mundo, no como una realidad estática, sino como una realidad en proceso, en transformación. En este escenario, los estudiantes perciben que el mundo influye en su modo de actuar y que a su vez sus acciones impactan en el entorno, por lo tanto, entienden que si ellos cambian, también lo hará el mundo (Dante, 2010).

Desde esta perspectiva filosófica que establece una relación de reciprocidad entre el sujeto y el objeto en el que ambos se transforman, será necesario que el docente vaya más allá en la comprensión de su realidad educativa y entienda la importancia de la relación que existe entre la filosofía y la pedagogía, al respecto Quintana (1982) establece que: "la pedagogía es el coronamiento y consumación de la filosofía" (p. 66). En este tenor, el razonamiento filosófico que desarrolle el docente se concretará en las acciones pedagógicas que emprenda dentro del aula.

En ese sentido, la formación de un nuevo maestro cobra mayor importancia, pues se trata de que su misma formación responda a las exigencias y demandas del siglo XXI en que es necesario que el profesor reconozca la relación entre la filosofía y la pedagogía, entendiendo a esta última como una práctica moral y política que trasciende el mero conocimiento teórico (Lucio et al, 2018), es decir, consiste en que el profesor comprenda que su actuar en el aula no se limita a la reproducción de un listado de estrategias de enseñanza aprendidas durante su formación inicial, sino que resulta en un ejercicio reflexivo de carácter ético, moral y político que impactará directamente en la realidad de los alumnos.

Las escuelas normales deben centrar su tarea en el desarrollo del pensamiento crítico de sus estudiantes a partir de los principios como los propuestos por Freire (1985), entre ellos destaca 
lo siguiente: concebir a la enseñanza como un acto creador y crítico, la necesidad de estudiar antes de enseñar, abordar distintos ámbitos desde lo local, regional, nacional y global, tomar distancia y ser curioso. Como se puede apreciar, la práctica educativa debe relacionar materiales, métodos y técnicas, en este sentido, el pensamiento crítico inquieta al educador y al educando, no frena su capacidad de pensar, favorece el diálogo pedagógico e implica tanto el contenido y objeto cognoscible como a los educandos quienes dialogan con el contenido; una enseñanza crítica considera como deber y derecho del docente enseñar en el tiempo y el espacio en el que se encuentra (Morales, en Lucio et al, 2018).

El pensamiento crítico que desarrollen los maestros les permitirá actuar desde una pedagogía crítica, lo que implica reflexionar y actuar de forma diferente a lo establecido, a partir de una comprensión del contexto y la realidad en la que se vive, donde se contempla la "justicia social, las minorías excluidas, la equidad de género, la eliminación de las brechas socioculturales. Es la búsqueda de una pedagogía alternativa, una pedagogía de la diferencia e involucrada en el movimiento de las nuevas perspectivas críticas hacia la educación” (Malagón y Rincón, 2018, p. 106).

En la pedagogía crítica existen tres elementos que el maestro debe priorizar para la formación de alumnos críticos, autónomos y libres: el primero de ellos consiste en comprender que tanto él, como sus alumnos son sujetos históricos que tienen un pasado, por tanto, el cambio de su realidad debe partir de la comprensión de su propia historia, a fin de que entiendan que su presente está en sus manos y que se comprometan con la mejora del futuro, no sólo individual, sino social (Lucio et al, 2018).

El segundo elemento que el docente debe considerar en la pedagogía crítica es el diálogo y la libertad de expresión, debido a que son el primer paso para generar un liderazgo transformador que verdaderamente conduzca al sujeto a pensar y reflexionar. No se trata de que el maestro convenza a los alumnos sobre una verdad, sino de escucharlos, aceptar sus ideas y juntos construir una nueva realidad que integre las posturas de los otros, sin priorizar ninguna. Se trata de integrar antes de imponer.

El tercer elemento es la unidad entre la acción y la reflexión sobre el mundo para transformarlo, lo que Freire reconoce como "praxis" (Lucio et al, 2018), la cual implica replantear la educación para alterar la realidad y el estado, al respecto Sánchez Vásquez (citado en Arriarán-Cuéllar, 2014) la relaciona con cuatro aspectos:

1. Una crítica de lo existente en nombre de la necesidad de justicia y libertad.

2. Un proyecto de emancipación (el socialismo como nueva sociedad).

3. Un conocimiento de la realidad a transformar ligado a una voluntad de cambio.

4. Por su voluntad de cambio, un vínculo con la practica adecuada al proyecto de emancipación. (pág. 153)

La praxis favorece la reflexión del sujeto a fin de transformar su entorno, de mejorar su realidad y de tener la voluntad de cambiar. Al desarrollar dicha praxis se puede superar la dualidad entre el opresor y el oprimido, para ello, se requiere que el profesor problematice a los estudiantes, los oriente hacia el cuestionamiento constante de las realidades impuestas por los más poderosos, los lleve a interrogar las normas, a poner en duda los mitos y a pensar en 
78 El papel del maestro en la transformación educativa ante un escenario de incertidumbre. Reflexiones desde y para México

alternativas de solución informadas que conduzcan a los estudiantes a diseñar nuevas propuestas de mejora.

La praxis también debe ser desarrollada por el profesor a fin de mejorar su práctica educativa. La formación de maestros está obligada a orientar a los docentes a la comprensión de su hacer en el aula como un objeto dinámico que demanda de un estudio y comprensión permanente. En este sentido, el profesor debe incorporar a sus actividades de enseñanza la investigación, pero una metodología que coincida con esta perspectiva y pedagogía crítica de la que hemos hablado, pues tendrá que incluirse él mismo como sujeto a estudiarse en lugar de solo investigar a los otros, como es el caso de la investigación-acción participativa (Lucio et al, 2018), de tal forma que desarrolle un proceso sistemático de estudio sobre su proceder desde un ejercicio colaborativo con sus alumnos para mejorar la realidad generada en el aula.

Sobre esta base, la comprensión que el profesor genere de su práctica no se limitará solo a su aula de clases, sino que debe expandir su horizonte al revisar la situación contextual en la que se encuentra, pues esto forma parte del pensamiento crítico, para ello, debe convertirse en un investigador curioso que interprete las cifras y haga comparaciones en el tiempo y el espacio, a fin de que desde su propia realidad pueda generar acciones para impactar en contextos de mayor amplitud.

En este sentido, un ejercicio que el docente debe realizar es la comparación de cifras que le ayuden a entender el nivel educativo en el que se encuentra, para identificar las limitaciones y sus fortalezas, lo que le permitirá diseñar acciones concretas para mejorar esta situación, una manera sencilla puede ser, revisar la situación de las escuelas normales en el estado de Zacatecas se observa en la tabla 1.

Tabla 1. Estadísticas sobre el número de estudiantes de las Escuelas Normales del Estado de Zacatecas

\begin{tabular}{|c|c|c|c|c|c|}
\hline Ciclo escolar & Total de alumnos & Primer grado & Segundo grado & Tercer grado & Cuarto grado \\
\hline 2016-2017 & 1668 & 460 & 441 & 366 & 390 \\
\hline 2017-2018 & 1,649 & 464 & 409 & 410 & 366 \\
\hline 2018-2019 & 1,743 & 537 & 420 & 370 & 416 \\
\hline 2019-2020 & 2,035 & Aún no hay dato & disponibles & & \\
\hline
\end{tabular}

La tabla uno refleja que la matrícula de alumnos de las escuelas normales en Zacatecas ha ido en aumento, lo que a simple vista podría resultar un logro, pues refleja un crecimiento paulatino del 22\% entre el ciclo 2016-2017 y el 2019-2020, lo que significaría un alcance favorable en la matrícula de la educación normal, pues cada vez abarca mayor cantidad de población; sin embargo, al hacer un seguimiento de la generación que ingresó en el ciclo escolar 2016-2017 a la escuela normal se observa un decremento: el primer grado se inició con 460 estudiantes, al segundo año redujo el 11\%, mientras que al tercero se disminuyó otro 9\%.Valdría la pena revisar las cifras del ciclo escolar 2019-2020 para valorar cuántos alumnos culminaron el cuarto año de la educación normal y cuántos lograron titularse, y de esa forma indagar a mayor profundidad los fenómenos educativos. 
Este tipo de ejercicios de análisis ayuda al maestro a comprender que su hacer en el aula no sólo impacta con el grupo de estudiantes que le fue asignado, sino que también forma parte de la realidad estatal, nacional e internacional, de esta forma, entenderá que sus acciones influyen en la transformación del mundo y la mejora de la realidad.

Ante un escenario incierto, en el que el docente juega un papel muy importante en el mejoramiento de la sociedad, conviene que no solamente revise cifras, sino que se interrogue sobre la función que desempeña en el plano mundial y nacional. En este sentido, puede también revisar acuerdos internacionales como por ejemplo, la Agenda 2030, específicamente el Objetivo de Desarrollo Sostenible número cuatro referente a "Garantizar una educación inclusiva y equitativa de calidad y promover oportunidades para el aprendizaje permanente para todos" (Organización de las Naciones Unidas, 2018, pág. 27), en la meta cuatro punto siete inciso "c" se habla de lo que se espera de la profesión docente, pues se propone que de aquí al 2030 se aumente considerablemente la oferta de docentes calificados, incluso mediante la cooperación internacional para la formación de docentes en los países en desarrollo, especialmente los países menos adelantados.

En el contexto internacional, el docente puede revisar la necesidad de que su formación responda a nuevos requerimientos para que se convierta en el promotor de las habilidades básicas referentes a la lectura y la escritura en estudiantes de educación obligatoria, en jóvenes y adultos, además de promover competencias técnicas que faciliten el manejo de las TIC. Ante un planteamiento internacional tan ambicioso y utópico, es necesario que el docente comprenda y participe de forma más activa en la conformación e implementación de las políticas nacionales, como es el caso de la NEM, que se autodefine como:

"La institución del Estado mexicano responsable de la realización del derecho a la educación en todo el trayecto de 0 a 23 años de edad de las y los mexicanos. Esta institución tiene como centro la formación integral de niñas, niños, adolescentes y jóvenes, y su objetivo es promover el aprendizaje de excelencia, inclusivo, pluricultural, colaborativo y equitativo a lo largo de su trayecto formativo" (Secretaría de Educación Pública, 2019, pág. 2)

Desde su concepción, se reconocen algunos principios del pensamiento crítico propuesto por Freire, como el aprendizaje inclusivo y pluricultural, el cual busca integrar las voces de las minorías y los oprimidos; además, se identifican aspectos de la pedagogía crítica como la equidad y la colaboración, en este sentido, la política educativa impulsada por la Nueva Escuela Mexicana brinda al docente la posibilidad de reflexionar y transformar su entorno.

Las condiciones que plantea la Nueva Escuela Mexicana son la asequibilidad (garantía del derecho a la educación), accesibilidad (educación gratuita para todos), aceptabilidad (criterios de seguridad, calidad y calidez como condiciones del profesorado) y adaptabilidad (adecuar la educación a los contextos). En este sentido, se observa la preocupación de la política educativa por tratar de adecuar la educación al contexto y asegurar este derecho en toda la población, lo que compromete al docente a reflexionar sobre su entorno y promover estrategias didácticas y de atención integral, que respondan a las necesidades de aprendizaje de sus alumnos en su situación real.

Los principios en los que se sustenta la NEM aseguran los fundamentos para la formación de un ciudadano libre, autónomo, con identidad nacional, responsabilidad social, inserto en una cultura de paz y un contexto intercultural. Uno de los principios que adquiere mayor relevancia en torno al tipo de profesor que demanda la sociedad del siglo XXI es la "participación para 
80 El papel del maestro en la transformación educativa ante un escenario de incertidumbre. Reflexiones desde y para México

la transformación de la realidad", es decir, desde la política educativa nacional se reconoce la necesidad de mejorar y cambiar los contextos para responder a las necesidades reales de los mexicanos, entonces, se requiere un profesor que logre generar en su ámbito de desempeño, esta nueva cultura social y educativa de transformación.

\section{Conclusión}

La transformación de la educación es una necesidad urgente que se presenta ante un escenario de incertidumbre, en este caso, evidenciado por la pandemia generada por la COVID-19, hecho que impulsó al Gobierno de la República a implementar estrategias que más que mantener al sistema educativo a flote, acentuaron las desigualdades sociales y reflejaron los viejos paradigmas desde los que se entiende el currículo como una lista rígida de contenidos que se deben revisar en un horario laboral dentro de la escuela. Se dejó de lado la función más importante de la educación referente a la formación de un individuo libre y autónomo, que esté habilitado para dar respuesta a un contexto real, toda vez que la escuela debe servir como medio pare reflexionar y mejorar su entorno.

Ante este escenario de incertidumbre es necesario formar al docente en una filosofía de la educación emergente para que reflexionen y comprendan su propia realidad, desde un sentido dinámico en el que su papel como educadores consiste en convertirse en agentes de cambio y transformación, es por ello que la sociedad actual demanda de una reforma permanente y reconstructiva de los currículos de las escuelas normales, a fin de que los profesores adquieran herramientas de mejora continua que les permitan pensar críticamente y analizar su realidad educativa desde diferentes perspectivas (cualitativas y cuantitativas), en distintas dimensiones tanto locales y estatales, como nacionales e internacionales.

La formación del docente que responda a los retos y exigencias del siglo XXI es una deuda pendiente con la sociedad. Las políticas educativas contenidas en la Nueva Escuela Mexicana tratan de saldarla, priorizando aspectos de la pedagogía crítica y favoreciendo la transformación social; sin embargo, este cambio no será posible en tanto que el magisterio no abandone el temor a la libertad y se siga concibiendo como una víctima del currículo, cuyo único remedio es seguir reproduciendo lo que ha aprendido, aunque esto lo convierta en un replicador de la violencia simbólica.

En países latinoamericanos como Cuba los profesionales de la educación se han atrevido a desarrollar propuestas sustentadas en la pedagogía crítica impulsada por Freire, en las que buscan mejorar el entorno rural con un enfoque de "síntesis cultural" (Lucio et al, 2018), es decir, estas propuestas innovadores tratan de abrazar la diversidad y promover un cambio progresista desde y para la propia comunidad, donde no se establece una visión hegemónica, sino que se reflexiona y dialoga con los demás para diseñar e implementar de forma conjunta propuestas que mejoren el entorno y la realidad de todos.

Estos antecedentes obligan a México a reflexionar en torno a sus modelos curriculares, tanto de educación básica, como en la formación docente, pues no es suficiente con que en el discurso oficial se incluyan ideas que buscan transformar la realidad, sino que este pensamiento debe ser asumido por los profesores y la población en general, a fin de que comprendan que el mejoramiento de la educación no es sólo la aplicación de un pensamiento hegemónico del gobierno, sino que, demanda de la participación de todos los ciudadanos en 
la conformación de una propuesta curricular sometida a una constante reflexión y análisis, orientada a atender las necesidades de las minorías y los oprimidos. En este sentido, esta contribución quisiera plantear una pregunta eje que se convierte en constante motor de generación y desempeño para los profesores de México ¿están dispuestos a transformar su entorno desde una reflexión filosófica y un pensamiento crítico?

\section{Referencias}

Arriarán-Cuéllar. (2014). Filosofía y praxis educativa según Adolfo Sánchez Vázquez. Universia, V (13), 143155.Dante (2010). Apuntes de catedra, filosofía de la educación. Prof. Cimaomo, http://www.scielo.org.mx/pdf/ries/v5n13/v5n13a9.pdf

Bauman, Z. (2008). Los retos de la educación en la modernidad líquida. Barcelona: Gedisa.https://www.uv.mx/mie/files/2012/10/retos-educacion-modernidad.pdf

Dante (2010). Apuntes de catedra, filosofía de la educación. Prof. Gabriel Cimaomo.

Delgado, B. G. (2020). Igualdad educativa y post pandemia. En Casanova, C. H. (Coord.). Educación y pandemia: una visión académica. (pp. 183-194) Universidad Nacional Autónoma de México, Instituto de Investigaciones sobre la Universidad y la Educación.

Enrique, D., \& Linares, R. (n.d.). FILOSOFÍA DE LA EDUCACIÓN. 1-50.

Freire, Paulo. (1985). Pedagogía del oprimido. Tierra Nueva. Siglo XXI Editores. México.

Fullat, O. G. (1987). Filosofía de la educación: conceptos y límites. Educar (11), 5-15.

IISUE (2020), Educación y pandemia. Una visión académica, México, unam, http:/ /www.iisue.unam.iisue/covid/educacion-y-pandemia, consultado 29 de abril, 2021.

Lucio, G. R., \& Cortez, Morales, A. (2018). Formación docente transformadora para liberar la educación. Paulo Freire y la Pedagogía del oprimido. En M. R. Páez, G. Rondón, H., \& J. Trejo, C. , Formación docente y pensamiento crítico en Paulo Freire (pág. 180). México: CLACSO.http://biblioteca.clacso.edu.ar/clacso/se/20181113025736/Formacion_docente_Paulo_Frei re.pdf

Organización de las Naciones Unidads. (2018). La Agenda 2030 y los Objetivos de Desarrollo Sostenible. Una oportunidad para América Latina y el Caribe. Naciones Unidas, Santiago: Naciones Unidas.https://repositorio.cepal.org/bitstream/handle/11362/40155/24/S1801141_es.pdf

Plá, S. (2020). La pandemia en la escuela:entre la opresión y la esperanza. En Casanova, C. H. (Coord.). Educación y pandemia: una visión académica. (pp. 30-38) Universidad Nacional Autónoma de México, Instituto de Investigaciones sobre la Universidad y la Educación.

Ratio, F. E. T. (2014). Filosofía de la Educación: Aproximación a un posible significado. PhilosophyofEducation: A possibleapproachtomeaning. Libardo Tristancho Calderón Instituto de Investigación en Ciencias de la Educación, Universidad la Salle. 8, 75-89. http://www.scielo.org.bo/scielo.php?script=sci_arttext\&pid=S2071-081X2014000200007

Secretaría de Educación Pública. (2019). La Nueva Escuela Mexicana: principios y orientaciones pedagógicas. México:

SEP.https://dfa.edomex.gob.mx/sites/dfa.edomex.gob.mx/files/files/NEM $\% 20$ principios $\% 20 \mathrm{y} \% 20$ orientacio $\% \mathrm{C} 3 \% \mathrm{ADn} \% 20$ pedago $\% \mathrm{C} 3 \%$ ADgica.pdf

Secretaría de Educación Pública. (2021). Principales cifras del sistema educativo nacional 2019-2020. México:

SEP. https://www.planeacion.sep.gob.mx/Doc/estadistica_e_indicadores/principales_cifras/principales_ci fras_2019_2020_bolsillo.pdf

Tünnerman, B. C. (2008). Panorama general sobre la filosofía de la educación. Nicaragua: La prensa S. Ahttps://www.sev.gob.mx/centros-rebsamen/files/2017/03/Panorama-general-sobre-la-filosofia-dela-e-educacion.pdf 